\title{
SUBLINGUAL IMMUNOTHERAPY IN THE TREATMENT OF ALLERGIC
} ASTHMA: A SYSTEMATIC REVIEW WITH META-ANALYSIS

Clin Biomed Res. 2017;37(4):334-340

1 Núcleo de Estudos em Saúde da Criança (NESC), Instituto de Pesquisas Biomédicas (IPB), Pontifícia Universidade Católica do Rio Grande do Sul (PUCRS). Porto Alegre, RS, Brazil.

2 Centro Universitário da Serra Gaúcha (FSG). Caxias do Sul, RS, Brazil.

Corresponding author:

Cristian Roncada

crisron@gmail.com

Núcleo de Estudos em Saúde

da Criança (NESC), Instituto de

Pesquisas Biomédicas (IPB), Pontifícia

Universidade Católica do Rio Grande do Sul (PUCRS)

Av. Ipiranga, 6681, Prédio 96A, sala 208. CEP 90619-900, Porto Alegre, RS, Brazil.

\section{Cristian Roncada ${ }^{1,2}$, Bianca Martininghi Bugança ${ }^{2}$, Karina Soldera², Paulo Márcio Pitrez ${ }^{1}$}

\section{ABSTRACT}

Introduction: The guidelines for the management of allergic respiratory diseases oriented towards control from medical treatment combined with measures of environmental hygiene. Immunotherapy is one of several types of treatment, applied in combination with prophylactic drugs and environmental care. The aim of the study is to evaluate the efficacy of sublingual immunotherapy (SLIT) for house dust mites (HDM) in people with allergic asthma.

Methods: The study is based on a systematic review with meta-analysis of randomized clinical trials, associating sublingual immunotherapy with the treatment of allergic patients with HDM.

Results: The searches were applied in PubMed, ScienceDirect and Scielo databases. Initially, 98 articles were recovered, of which only nine were eligible. Of these, eight $(88.9 \%)$ were conducted in Europe and only one (11.1\%) in Asia. Comparing the outcomes expiratory volume in the first minute $\left(\mathrm{FEV}_{1}\right)$ and sensitivity to allergens (HDM) between SLIT and placebo groups before and after intervention, no differences were observed between the groups.

Conclusions: SLIT is not evidenced significantly by meta-analysis for the treatment of allergic asthma.

Keywords: Mites; asthma; rhinitis; respiratory disease

Allergic respiratory diseases, particularly asthma, have a high prevalence ${ }^{1}$, showing a significant increase in recent decades, both in the pediatric and adult populations ${ }^{2}$, and may manifest with perennial or seasonal symptoms ${ }^{3}$. Perennial manifestations are the ones more commonly observed and are associated with sensitivity to household allergens ${ }^{4}$. House dust mites (HDM) allergens are the major triggering factors for the aggravation of these diseases, followed by other household allergens such as animal epithelia, fungi, and cockroach $^{5}$. The treatment of these allergic diseases combines drug therapy with immunotherapy and environmental prophylaxis ${ }^{6}$.

Allergen-specific immunotherapy consists of administering gradually increasing small doses of allergens over months or years and promotes a long-lasting effect even after treatment discontinuation ${ }^{7}$. Despite advances in the understanding of imunopathogenesis and pathophysiology of allergic respiratory diseases and in the development of efficient drugs to control airway inflammation and associated symptoms, so far immunotherapy, along with environmental hygiene practices, seems to be the only therapeutic strategy that managed to modify the natural progression of allergic respiratory diseases by promoting their improvement or even their remission and to prevent their worsening and the development of new sensitization events, exhibiting long-lasting effects even after immunotherapy discontinuation ${ }^{8}$.

Allergen-specific immunotherapy should only be considered when the profile of specific-IgE sensitization is identified, preferably by immediate reading skin tests or, alternatively, by specific IgE testing with a radioallergosorbent test 
(RAST), and when symptoms are clearly related to exposure to the identified allergens ${ }^{9}$. Patients with allergic rhinitis or allergic asthma whose symptoms are not adequately controlled despite optimized therapy and environmental control and those whose disease control requires high drug doses or a combination of multiple drugs are potential candidates for immunotherapy, as well as those who develop adverse drug effects or those who want to prevent these effects ${ }^{10}$.

The efficacy of immunotherapy has been recently demonstrated by randomized clinical trials, due to the availability of allergen extracts increasingly more purified and standardized with regard to their efficacy, which allowed us to accurate define antigen doses administered during immunotherapy ${ }^{11}$. There are two types of immunotherapy for the treatment of allergic respiratory diseases: subcutaneous immunotherapy (SCIT) and sublingual immunotherapy (SLIT). SCIT is the most widely used worldwide and consists of the subcutaneous administration of allergen doses. Although this technique has good efficacy, it may have limited adherence, especially in the pediatric population ${ }^{12}$. Conversely, SLIT does not have the same limitations regarding treatment adherence; however, evidence of its efficacy is still controversial ${ }^{13}$. SLIT has been increasingly used in Europe but not in countries like USA, where it is still under investigation, since no formulation has been approved yet by the Food and Drug Administration (FDA) ${ }^{14}$.

In view of these findings, the present systematic review aimed to assess the most relevant studies that compared SLIT against HDM allergens with placebo in patients with allergic asthma.

\section{METHODS}

This study consisted of a systematic review with meta-analysis of three health databases to identify the most relevant double-blind randomized clinical trials that compared a group receiving SLIT against HDM allergens with another receiving placebo.

\section{Inclusion Criteria}

This review included double-blind randomized clinical trials investigating SLIT for the treatment of allergic asthma induced by HDM. Articles that did not have a placebo group, narrative reviews, systematic reviews, meta-analyses, case reports, and guidelines were excluded from the present review.

\section{Search Strategy}

The search was performed using specific descriptors English language, using the boolean operators $A N D$ and $O R$. Parentheses were used to indicate a group of search terms and quotation marks (") to identify compound words. Therefore, the following search formula was used: (("Sublingual Immunotherapy" OR SLIT) AND (Asthma) AND ("House Dust Mites" OR $H D M)$. Searches were made in PubMed (Medline), ScienceDirect (Elsevier) and Scielo (Bireme) in October 2016 , with no restrictions on year of publication.

To avoid the inclusion of an excessive number of articles, searches were limited to title, keywords, and abstract fields. Thus, descriptors must be present in at least one of the search fields. Additionally, no search filters were specified, such as language, target audience, or type of study.

Articles selected from databases were then exported to a software for managing systematic reviews called State of the Art through Systematic Review (StArt) ${ }^{15}$, which helped to identify duplicate, excluded, and included articles. These analyses were conducted separately by two researchers (BMB, KS) and analyzed by a third researcher (CR).

Article eligibility was based on three inclusion and exclusion criteria: a) articles selected by the two investigators were included; b) articles not selected by either of the two investigators were excluded; c) articles selected by only one investigator were assessed by the reviewer and were included if meeting inclusion criteria.

After eligible articles were selected and outcome variables were identified, the Review Manager (RevMan $)^{16}$ was used and differential bivariate means (between group estimates - case vs. placebo), with a $95 \%$ confidence interval $(95 \% \mathrm{Cl})$ to estimate means for forced expiratory volume in the first second $\left(\mathrm{FEV}_{1}\right)$ and skin prick test (SPT) before and after intervention.

This systematic review was registered in the Centre for Reviews and Dissemination (PROSPERO) database ${ }^{17}$ under number CRD42015023199.

\section{RESULTS}

Initially, 98 articles were retrieved from databases (57 from PubMed, 36 from ScienceDirect, and five from Scielo). Of these, 17 articles published in duplicate were excluded, as well as 62 articles that did not include a placebo group or were not related to the proposed topic. Therefore, 19 eligible articles were pre-selected for full reading, of which 10 were excluded because they did not meet eligibility criteria, 
resulting in nine articles included in the meta-analysis, as shown in the flowchart below (Figure 1).

Of the nine studies included, eight $(88.9 \%)$ were conducted in Europe and only one (11.1\%) were conducted in Asia. Overall, seven articles $(77.7 \%)$ were published in the last 5 years (Table 1 ). Table 2 describes the main characteristics of study participants and outcome variables analyzed in the meta-analysis. Figures 2 and 3 show a comparison of sensitization to HMD and FEV1 before and after intervention. For both outcomes, no significant differences were observed between groups that received SLIT and those that received placebo immunotherapy.

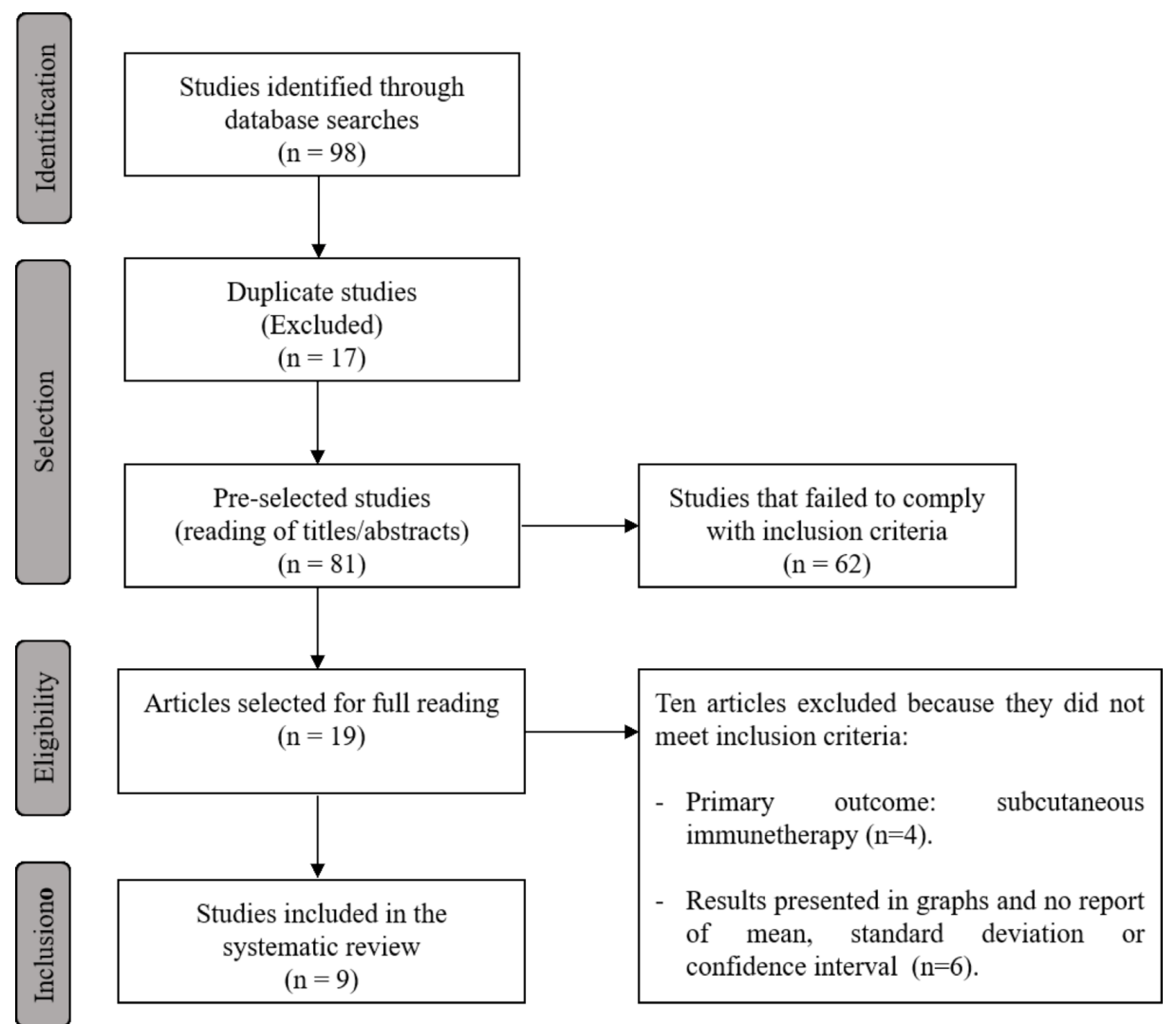

Figure 1: Flowchart of eligible articles and those included in the meta-analysis.

Table 1: General characteristics of eligible studies included in the systematic review.

\begin{tabular}{|c|c|c|c|c|c|c|c|c|}
\hline Author & Year & Country & Diagnostic test & Reagents $^{\dagger}$ & Disease & Intervention & Sensitization & Type \\
\hline Aydogan et al. ${ }^{18}$ & 2013 & Turkey & SPT & 20 & Asthma & SLIT & HMD & DP/DF $1: 1$ \\
\hline Bahçeciler et al. ${ }^{19}$ & 2001 & Turkey & SPT & 2 & Asthma & SLIT & HMD & DP/DF 1:1 \\
\hline Bousquet et al. ${ }^{20}$ & 2001 & France & SPT & 20 & Asthma & SLIT & HMD & DP/DF 1:1 \\
\hline Corzo et al. ${ }^{* 21}$ & 2014 & Spain & SPT & 2 & Asthma & SLIT & HMD & DP/DF $1: 1$ \\
\hline Corzo et al. ${ }^{* * 21}$ & 2014 & Spain & SPT & 2 & Asthma & SLIT & HMD & DP/DF $1: 1$ \\
\hline Marogna et al. ${ }^{22}$ & 2010 & Italy & RAST & 2 & Asthma & SLIT & HMD & DP/DF 1:1 \\
\hline Pham-Thi et al. ${ }^{23}$ & 2007 & France & SPT/RAST & 9 & Asthma & SLIT & HMD & DP/DF $1: 1$ \\
\hline Wang et al. ${ }^{24}$ & 2013 & China & SPT & 2 & Asthma & SLIT & HMD & DP/DF $1: 1$ \\
\hline Yukselen et al. ${ }^{25}$ & 2012 & Turkey & SPT & 2 & Asthma & SLIT & HMD & DP/DF 1:1 \\
\hline Yukselen et al. ${ }^{26}$ & 2013 & Turkey & SPT & 2 & Asthma & SLIT & HMD & DP/DF $1: 1$ \\
\hline
\end{tabular}

†Number of reagents assessed on diagnosis; *Study conducted with adults; ${ }^{*}$ Study conducted with children; SPT: skin prick test; RAST: radioallergosorbent test; SLIT: sublingual immunotherapy; HMD: house dust mites; DP: Dermatophagoide pteronyssinus; DF: Dermatophagoide farinae; $1: 1: 50 \%$ for each specimen. 
Table 2: Mean and standard deviation of studies included in this systematic review.

\begin{tabular}{|c|c|c|c|c|c|c|}
\hline & \multicolumn{3}{|c|}{ Sublingual immunotherapy } & \multicolumn{3}{|c|}{ Placebo immunotherapy } \\
\hline & $\mathbf{N}$ & $\%$ & Mean $\pm S D$ & $\mathbf{N}$ & $\%$ & Mean士SD \\
\hline Sex (male) & $322 / 621$ & 51.9 & & $187 / 351$ & 53.3 & \\
\hline Age (5-63 years) & & & $16.2 \pm 4.6$ & & & $16.4 \pm 4.4$ \\
\hline Duration of symptoms (months) & & & $67.0 \pm 40.4$ & & & $59.0 \pm 38.1$ \\
\hline Total score of asthma symptoms & & & $2.8 \pm 1.6$ & & & $2.1 \pm 1.1$ \\
\hline Total score of medication use & & & $0.2 \pm 0.03$ & & & $0.1 \pm 0.02$ \\
\hline Metalcolina $\mathrm{PC}_{20}(\mathrm{mg} / \mathrm{mL})$ & & & $1.1 \pm 0.8$ & & & $1.8 \pm 0.7$ \\
\hline \multicolumn{7}{|l|}{ Sensitivity to DP (mm) } \\
\hline Pre- treatment & & & $6.3 \pm 2.5$ & & & $5.8 \pm 2.4$ \\
\hline Post- treatment & & & $4.0 \pm 1.8$ & & & $6.5 \pm 2.8$ \\
\hline \multicolumn{7}{|l|}{ Sensitivity to DF (mm) } \\
\hline Pre- treatment & & & $6.2 \pm 3.3$ & & & $6.1 \pm 3.4$ \\
\hline Post- treatment & & & $3.9 \pm 2.5$ & & & $5.6 \pm 2.0$ \\
\hline \multicolumn{7}{|l|}{$\mathrm{FEV}_{1}(\%$ predicted $)$} \\
\hline Pre-treatment & & & $89.5 \pm 13.56$ & & & $84.5 \pm 14.0$ \\
\hline Post-intervention & & & $95.0 \pm 12.4$ & & & $96.3 \pm 13.0$ \\
\hline \multicolumn{7}{|l|}{ PEF (\% predicted) } \\
\hline Pre- treatment & & & $84.3 \pm 17.0$ & & & $89.3 \pm 21.8$ \\
\hline Post- treatment & & & $98.4 \pm 21.8$ & & & $93.7 \pm 17.8$ \\
\hline
\end{tabular}

DP: Dermatophagoide pteronyssinus; DF: Dermatophagoide farinae; $\mathrm{FEV}_{1}$ : forced expiratory volume in the first second; PEF: peak expiratory flow.

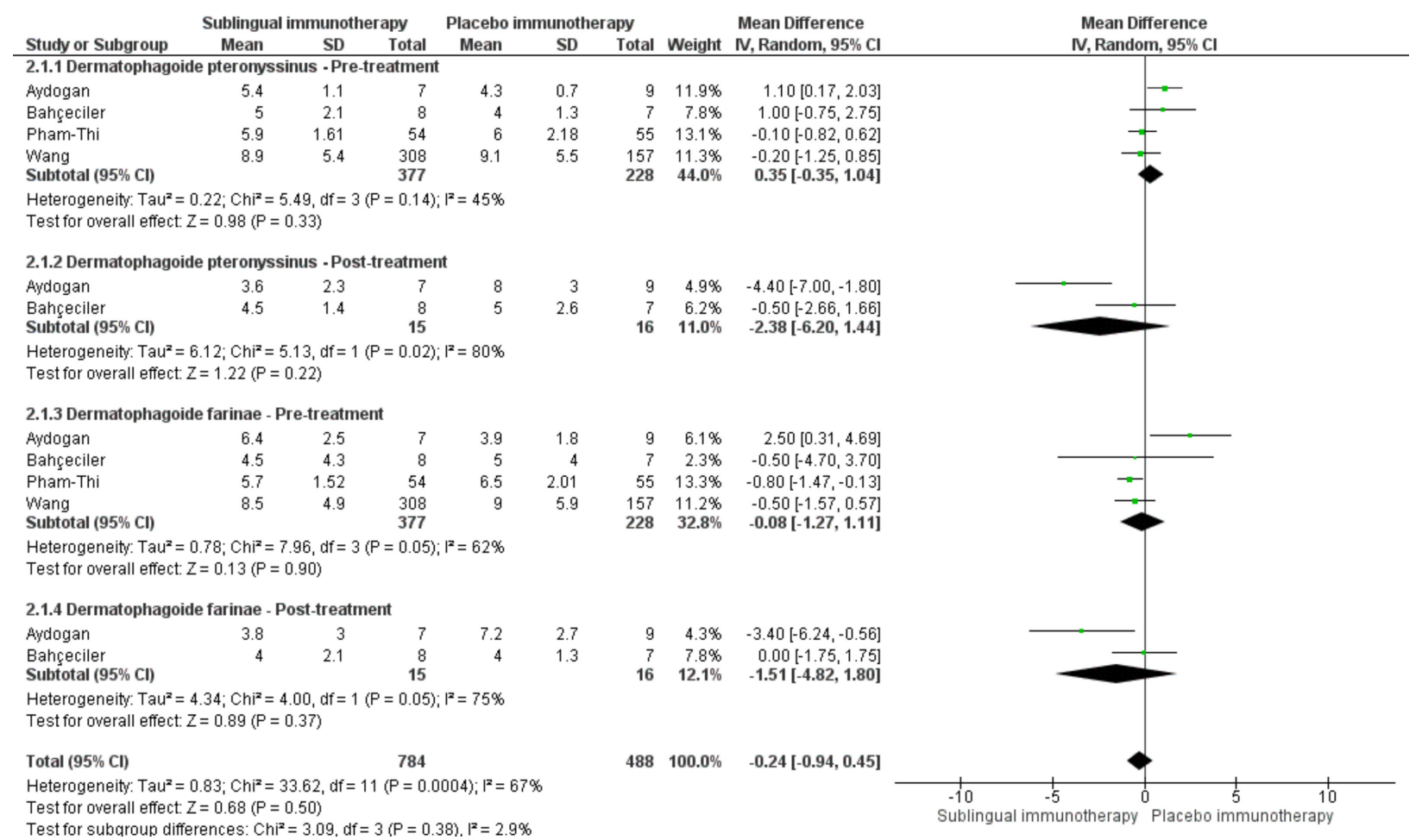

Figure 2: Difference in sensitization to Dermatophagoide pteronyssinus and Dermatophagoide farinae before and after SLIT in groups receiving SLIT and placebo. 


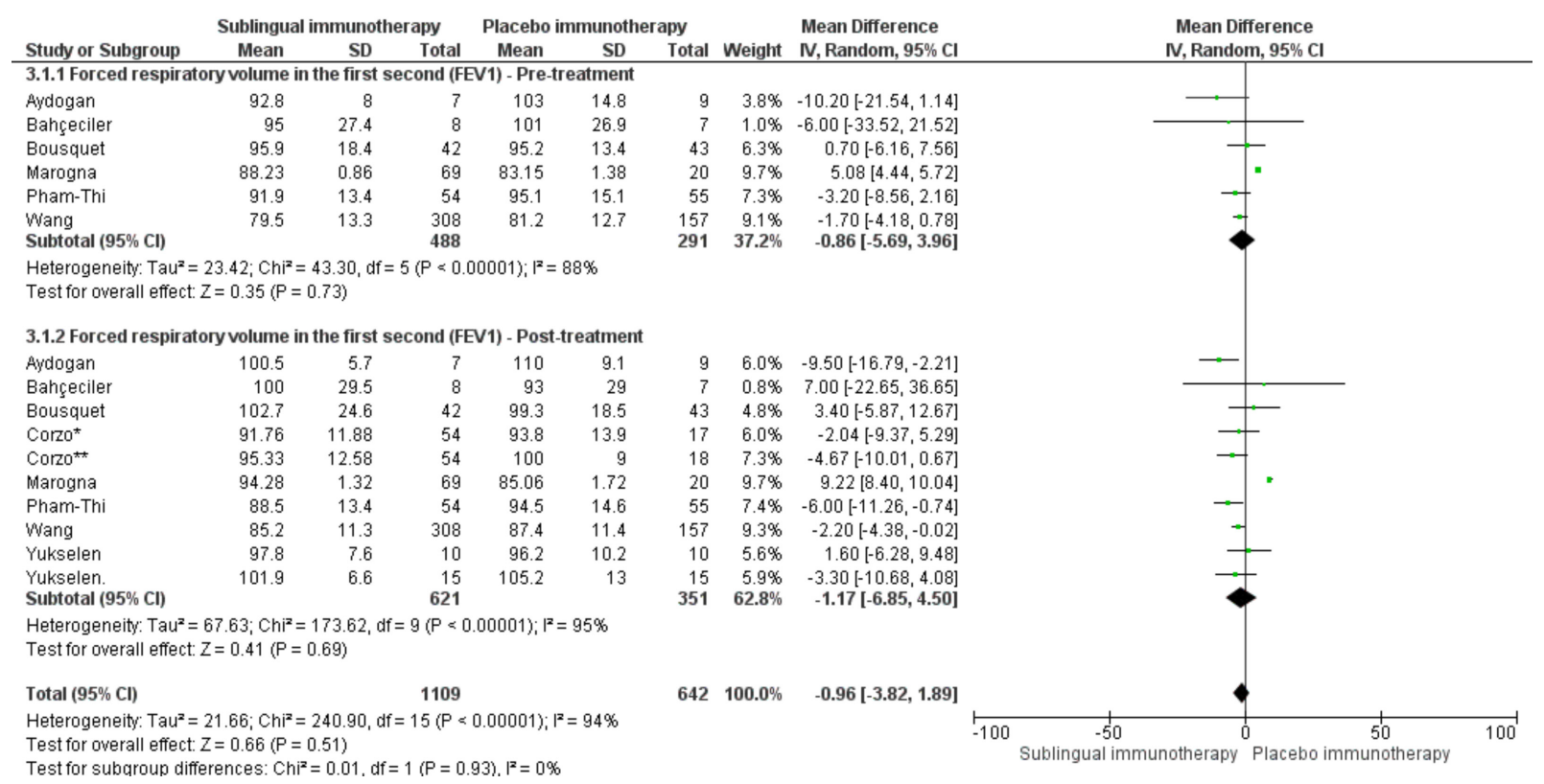

Figure 3: Difference in forced respiratory volume in the first second before and after SLIT in groups receiving SLIT and placebo.

\section{DISCUSSION}

Evidence on the efficacy of SLIT has been subject of debate ${ }^{27}$, being considered a supportive treatment with little efficacy in the management of allergic respiratory diseases. Additionally, studies suggest that SLIT is not effective either for all allergens or for all patients, which hindered its acceptance in the scientific community with regard to its applicability. ${ }^{28}$ As previously described by international guidelines ${ }^{27,28}$ for the management of respiratory diseases, the present meta-analysis did not find significant evidence on the efficacy of HDM sensitivity by SLIT in the treatment of allergic asthma. Although some studies found satisfactory values when using ITSL with HMD in the same study population, means for outcomes variables were not statistically different between groups receiving SLIT and placebo.

No intra or inter-group differences in sensitization to HMD Dermatophagoide pteronyssinus and Dermatophagoide farine were observed $\left(\mathrm{Chil}^{2}=3.09\right.$, df 3, $p=0.38, I^{2}=2.9 \%$ ) between SLIT and placebo groups before and after intervention. Similarly, no differences were observed in $\mathrm{FEV}_{1}\left(\mathrm{Chil}^{2}=0,01\right.$, df $1 \mathrm{p}=0,93, \mathrm{l}^{2}=0 \%$ ).

In the study by Aydogan et al. ${ }^{18}$, after 12 months of treatment, no significant differences were observed in the within and between-group comparisons of SLIT and groups based on disease symptom score and drug use score $(p>0.05)$. Skin reactivity to Dermatophagoides pteronyssinus was significantly reduced in SLIT compared to placebo group $(p=0.018)$.
Additionally, a significant reduction in nasal sensitivity was observed in SLIT group after one year treatment when compared to baseline $(p=0.04)$. The proportion of patients with non-specific bronchial hyperreactivity had an almost 3-fold increase in placebo group compared to baseline. The study by Aydogan et al. ${ }^{18}$ concluded that -SLIT with HDM has a modulating effect on allergen-specific nasal sensitivity, skin reactivity, and bronchial responsiveness but is not superior to placebo in reducing rhinitis symptoms.

Bahçeciler et al. ${ }^{19}$ found similar results to those by Aydogan et al. ${ }^{18}$ with regard to sensitization to HMD. At the end of the study, there was a reduction in the use of nasal corticosteroids to control rhinitis symptoms in the SLIT group compared with the placebo group $(p=0.004)$. Baseline skin sensitivity to $D$. pteronyssinus and $D$. farinea was not significantly different between in the two groups At the end of 6 months, PEF values in the placebo group were significantly lower than in the SLIT group $(p=0.049)$. Throughout the treatment period, the SLIT group was found to have less asthma exacerbations than the placebo group $(p=0.007)$. Results of this study suggests that SLIT may be a useful complementary therapy for respiratory diseases. Bousquet et al. ${ }^{20}$ showed that there was a moderate difference in in daytime asthma scores and increased sensitization to methacholine in SLIT group compared with placebo group. Additionally, the authors found that use of inhalers was significantly decreased after 25 months of treatment in both groups. 
Corzo et al. ${ }^{21}$ conducted a study with two different placebo-controlled trials (trial 1: adults; trial 2: children) and observed immunological changes and $\operatorname{lgE}$ blockade in a dose-dependent manner in both trials. The doses used in these trial were were tolerated in the selected populations, and thus were found found to be suitable for further clinical investigations in adults and children with allergic diseases.

Pham-Thi et al. ${ }^{23}$ conducted a study to assess the efficacy of SLIT in 111 childran and adolescents aged from 5 to 15 years with HMD-induced mild to moderate asthma. After a 4-week baseline phase, patients were randomly assigned to receive SLIT with tablets of HDM extract $(n=55)$ or placebo $(n=56)$ for 18 months. Pharmacologic treatment was adjusted every 3 months. Asthma symptom scores, reduction in use of inhaled corticosteroids and inhaled beta $_{2}$-agonists, rhinitis symptoms, lung function tests, and skin sensitivity to HDM were assessed during the study. After 18 months of treatment, diurnal and nocturnal asthma symptoms scores did not show significant differences between SLIT and placebo groups. Inhaled beta ${ }_{2}$-agonist was reduced in both groups without significant differences between groups. There were no significant differences in lung function $\left(F E V_{1}\right)$ and peak flow rate variations between groups. Rhynitis symtom score decreased in both groups, with no difference between the two groups. SLIT induced a significant reduction of skin sensitivity to HDM $(p<0.01)$. However, the authors report that SLIT does not provide additional benefit for children with asthma.

Wang et al. ${ }^{24}$ developed a study with 484 asthmatic adults over 12 months of treatment with SLIT and placebo. The proportion of asthmatic patients who exhibited SLIT response was low, with no significant difference with placebo group. For patients with moderate asthma $(n=175)$, treatment success rate greater in the SLIT group than in the placebo group (80.5\% and 66.1 , respectively; $p=0.021)$. No significant difference was observed in patients with mild asthma $(n=237)$. However, in the subgroup of patients with moderate asthma, the percentage of patients with totally controlled asthma was greater $(p=0.008)$ in SLIT group compared with placebo group (54.0\% and $33.9 \%$, respectively). However, the author suggest that sensitization with SLIT in large groups of patients with moderate persistent asthma may be efficient and safe to reach asthma control when combined with steroid therapy.

Yukselen et al. ${ }^{25}$ evaluated the efficacy of SLIT and SCIT with HMD in children with allergic rhinitis and asthma compared with placebo. After 12 months of treatment, SCIT was found to be more effective in reducing disease symptoms compared with placebo and SLIT. However, nasal provocative doses increased in both treatment groups (SLIT and SCIT) compared with placebo group. Nasal eosinophil after nasal challenge decreased with two treatment modes compared with baseline, and the clinical efficacy of SCIT and SLTI on rhinitis and asthma symptoms was more evident when compared with the placebo after 12 months of treatment.

Yukselen et al. ${ }^{26}$ published a study expanding the previous one and using the same criteria. The main findings of this study showed that clinical and immunological parameters were decreased since the first year of SCIT and SLIT and were maintained in the second year of the study, The study show that clinical immunological improvement with SCIT starts after the first years of immunotherapy, requiring more prolonged treatment with SLIT in children with rhinitis and asthma. However, HMD sensible children receiving SCIT have better response to hyperreactivity that children receiving SLIT.

Therefore, we concluded that the efficacy of SLIT in the treatment of allergic diseases could not be consistently confirmed be by meta-analysis. Additionally, the studies analyzed showed that there was a slight difference between SLIT and placebo groups; however, to reach a minimally acceptable efficiency, the treatment should be implement for more than 24 months, hindering treatment adherence.

\section{REFERENCES}

1. Mallol J, Crane J, von Mutius E, Odhiambo J, Keil U, Stewart A. The International Study of Asthma and Allergies in Childhood (ISAAC) Phase Three: a global synthesis. Allergol Immunopathol. 2013;41:7385.

2. Ozdoganoglu T, Songu M. The burden of allergic rhinitis and asthma. Ther Adv Respir Dis. 2012;6:11-23.
3. Smith H, Horney D, Goubet S, Jones C, Raza A, White P, et al. Pragmatic randomized controlled trial of a structured allergy intervention for adults with asthma and rhinitis in general practice. Allergy. 2015;70:203-11.

4. Hamann D, Hamann CR, Thyssen JP. The impact of common metal allergens in daily devices. Curr Opin Allergy Clin Immunol. 2013:13:525-30.
5. Araujo LM, Rosario NA, Mari A. Molecular-based diagnosis of respiratory allergic diseases in children from Curitiba, a city in Southern Brazil. Allergol Immunopathol. 2016;44:18-22.

6. Ring J, Akdis C, Lauener R, Schäppi G, Traidl-Hoffmann C, Akdis $\mathrm{M}$, et al. Global Allergy Forum and Second Davos Declaration 2013 
Allergy: barriers to cure-challenges and actions to be taken. Allergy. 2014;69:978-82.

7. Cox L, Wallace D. Specific allergy immunotherapy for allergic rhinitis: subcutaneous and sublingual. Immunol Allergy Clin North Am. 2011;31:561-99.

8. Chong Neto HJ, Rosário NA, Solé D. Asthma and rhinitis in South America: how different they are from other parts of the world. Allergy Asthma Immunol Res. 2012;4:62-7.

9. Tripodi S, Frediani T, Lucarelli S, Macrì F, Pingitore G, Di Rienzo Businco A, et al. Molecular profiles of IgE to Phleum pratense in children with grass pollen allergy: implications for specific immunotherapy. J Allergy Clin Immunol. 2012;129:834-9.

10. Bousquet J, Schünemann $\mathrm{HJ}$, Samolinski B, Demoly P, BaenaCagnani CE, Bachert C, et al. Allergic Rhinitis and its Impact on Asthma (ARIA): achievements in 10 years and future needs. J Allergy Clin Immunol. 2012;130:1049-62.

11. Varshney P, Jones SM, Scurlock AM, Perry TT, Kemper A, Steele P, et al. A randomized controlled study of peanut oral immunotherapy: clinical desensitization and modulation of the allergic response. J Allergy Clin Immunol. 2011;127:654-60.

12. Tabatabaian F, Casale TB. Selection of patients for sublingual immunotherapy (SLIT) versus subcutaneous immunotherapy (SCIT). Allergy Asthma Proc. 2015;36:100-4.

13. Dretzke J, Meadows A, Novielli N, Huissoon A, Fry-Smith A, Meads C. Subcutaneous and sublingual immunotherapy for seasonal allergic rhinitis: a systematic review and indirect comparison. J Allergy Clin Immunol. 2013;131:1361-6.

14. Burks AW, Calderon MA, Casale T, Cox L, Demoly P, Jutel M, et al. Update on allergy immunotherapy: American Academy of Allergy, Asthma
\& Immunology/European Academy of Allergy and Clinical Immunology/ PRACTALL consensus report. $J$ Allergy Clin Immunol. 2013;131:128896.e3.

15. Zamboni A, Di Thommazo A, Hernandes E, Fabbri S, editors. StArt: uma ferramenta computacional de apoio à revisão sistemática. In: Anais do Congresso Brasileiro de Software (CBSoft'10); 2010; Salvador, Brazil. 2010.

16. The Nordic Cochrane Centre. The Cochrane Collaboration. Review Manager (RevMan). 2011. 5 p.

17. Centre for Reviews and Dissemination. PROSPERO: International prospective register of systematic reviews. York. [cited 2017 June 20]. Available from: http://www. crd.york.ac.uk/PROSPERO

18. Aydogan M, Eifan AO, Keles S, Akkoc T, Nursoy MA, Bahceciler NN, et al. Sublingual immunotherapy in children with allergic rhinoconjunctivitis mono-sensitized to house-dust-mites: a double-blind-placebo-controlled randomised trial. Respir Med. 2013;107:1322-9.

19. Bahçeciler NN, Işik U, Barlan IB, Başaran MM. Efficacy of sublingual immunotherapy in children with asthma and rhinitis: a double-blind, placebo-controlled study. Pediatr Pulmonol. 2001;32:49-55.

20. Bousquet J, Scheinmann $P$, Guinnepain MT, Perrin-Fayolle M, Sauvaget J, Tonnel AB, et al. Sublingual-swallow immunotherapy (SLIT) in patients with asthma due to house-dust mites: a double-blind, placebo-controlled study. Allergy. 1999:54:249-60.

21. Corzo JL, Carrillo T, Pedemonte C, Plaza Martin AM, Martín Hurtado $\mathrm{S}$, Dige $\mathrm{E}$, et al. Tolerability during double-blind randomized phase I trials with the house dust mite allergy immunotherapy tablet in adults and children. J Investig Allergol Clin Immunol. 2014;24:154-61.
22. Marogna M, Colombo F, Cerra C, Bruno M, Massolo A, Canonica GW, et al. The clinical efficacy of a sublingual monomeric allergoid at different maintenance doses: a randomized controlled trial. Int J Immunopathol Pharmacol. 2010;23:937-45.

23. Pham-Thi N, Scheinmann P, Fadel R, Combebias A, Andre C. Assessment of sublingual immunotherapy efficacy in children with house dust miteinduced allergic asthma optimally controlled by pharmacologic treatment and mite-avoidance measures. Pediatr Allergy Immunol. 2007;18:47-57.

24. Wang L, Yin J, Fadel R, Montagut A, de Beaumont O, Devillier P. House dust mite sublingual immunotherapy is safe and appears to be effective in moderate, persistent asthma. Allergy. 2014;69:1181-8.

25. Yukselen A, Kendirli SG, Yilmaz M, Altintas DU, Karakoc GB. Effect of one-year subcutaneous and sublingual immunotherapy on clinical and laboratory parameters in children with rhinitis and asthma: a randomized, placebo-controlled, double-blind, double-dummy study. Int Arch Allergy Immunol. 2012;157:28898.

26. Yukselen A, Kendirli SG, Yilmaz M, Altintas DU, Karakoc GB. Two year follow-up of clinical and inflammation parameters in children monosensitized to mites undergoing subcutaneous and sublingual immunotherapy. Asian Pac J Allergy Immunol. 2013;31:23341.

27. British Thoracic Society Scottish Intercollegiate Guidelines Network. British guideline on the management of asthma. Thorax. 2008;63(Suppl 4):iv1-121.

28. British Thoracic Society. British guideline on the management of asthma. Thorax. 2014;69(Suppl 1):1192. 